\title{
Risk analysis of extended reach wells in the Liuhua Oilfield, South China Sea, based on comprehensive fuzzy evaluation method
}

\author{
Zhang Hui*, Gao Deli and Hao Zhiwei \\ MOE Key Laboratory of Petroleum Engineering, China University of Petroleum, Beijing 102249, China
}

\begin{abstract}
Drilling engineering has great uncertainty and it always involves huge investment and high risk. Risk analysis of extended reach drilling (ERD) is very important to prevent complex failures and to improve drilling efficiency. Nowadays there are few reports on how to analyze quantitatively the drilling risk for extended reach wells (ERWs). Based on the fuzzy set theory, a comprehensive fuzzy evaluation model for analyzing risks of ERD is proposed in this paper. Well B6ERW07 is a planned 8,000-meter ERW with a high ratio of horizontal displacement (HD) to vertical depth (VD) in the Liuhua Oilfield, the South China Sea, China. On the basis of the evaluation model developed in this study, the risk for drilling Well B6ERW07 was evaluated before drilling. The evaluation result shows that the success rate of drilling this well is predicted to be $51.9 \%$, providing important rational and scientific information for the decisionmakers.
\end{abstract}

Key words: Extended reach well, risk analysis, comprehensive fuzzy evaluation, weight value

\section{Introduction}

Extended reach drilling (ERD), a general expression for controlled directional well, horizontal well, deep well, and ultra-deep well technologies, is currently the world's most advanced drilling technology. Since the 1990s, with the extensive use of ERD technology, researchers and engineers have developed several programs to calculate friction, torque, and hydraulic parameters, and invented such drilling tools as guide motor, measurement while drilling (MWD), logging while drilling (LWD), controllable tapered stabilizer, and float casing appliances to improve greatly the success rate of drilling an extended reach well (ERW) (Jiang, 1999). However, when drilling an ERW, there are still many uncertain risks, which sometimes lead to drilling failures. Understanding various drilling risks during ERD and forecasting possible drilling failures have been the common concern of more and more drilling contractors and drillers.

Nowadays there are few reports on how to analyze quantitatively the risks of ERD. This paper analyzed comprehensively the multi-factor risks of ERD on the basis of fuzzy evaluation. A model was established for evaluating the extent of the risk of ERD, and the solution to the model was obtained using the Delphi method. Finally a quantitative risk analysis of Well B6ERW07, an 8,000-meter ERW with a high HD to VD ratio in the Liuhua LH11-1 Oilfield, South China Sea, China, was made.

*Corresponding author. email: zhanghui3702@163.com

Received December 18, 200

\section{Establishment of risk evaluation model for ERD}

Through investigating various major risk factors of ERD, we established a model for evaluating ERD risk, as shown Fig. 1. The risk is made up of two parts. One is risk of control before drilling and during drilling operation. Another is risk of equipment and its operation. Risk of control before drilling and during drilling operation is related to multiple key techniques of ERD, including 9 evaluation indices. Risk of equipment and its operation is made up of driller operational level and drill rig ability (Modi and Mason, 1997).

\section{Data acquisition of evaluation indices}

\subsection{Introduction to Delphi method}

The Delphi method, also called the expert investigation method, is an interactive forecasting method which relies on a panel of independent experts. The carefully selected experts answer questionnaires in two or more rounds. After each round, a facilitator provides an anonymous summary of the experts' forecasts from the previous round as well as the reasons they provided for their judgments. Thus the experts are encouraged to revise their earlier answers in light of the replies of other members of their panel. It is believed that during this process the range of the answers will decrease and the group will converge towards the "correct" answer. Finally, the process is stopped after a pre-defined stop criterion (e.g. number of rounds, achievement of consensus, and stability of results) and the mean or median scores of the final rounds determine the results (Wang, 2000; Zhang and Zhang, 1999). 


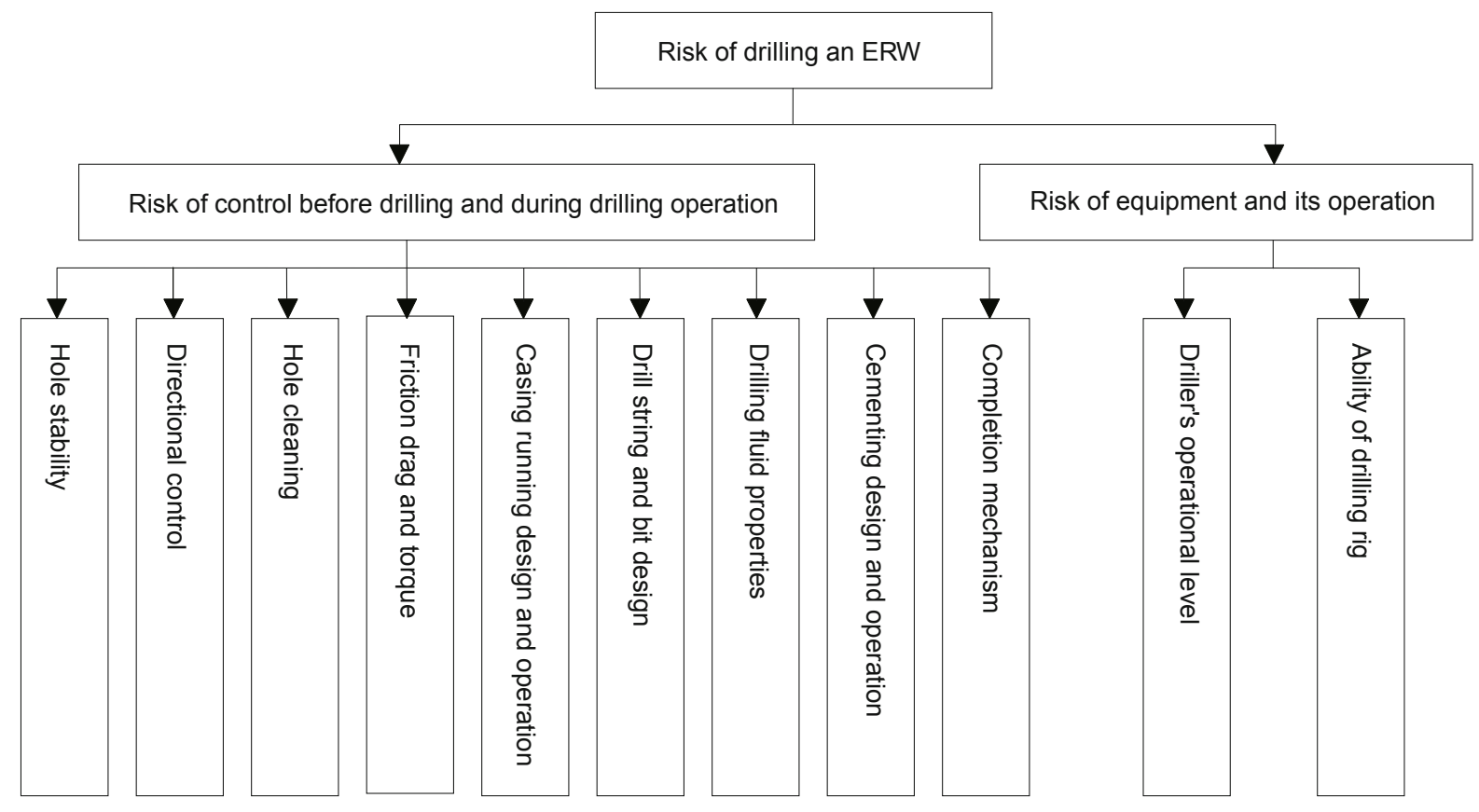

Fig. 1 Risk evaluation model for ERD

\subsection{Data acquisition}

The key of comprehensive evaluation for an ERW is to acquire two types of data: the weights of evaluation indices (risk factors) of the risk evaluation model mentioned above and grading data about risk of each evaluation index. According to the fundamental principle of Delphi method, both classes of data are acquired from a questionnaire survey.

\subsubsection{Determination of weights of risk evaluation indices}

The weighting factor decision method was used to ascertain the weight of each index, and the procedure is as follows (Zhang and Gao, 2005):

(1) Select a panel of experts.

(2) Design a well-structured questionnaire concerning the weighting factors of risk factors, as shown in Table 1.

Table 1 Point table about obtaining weighting factors

\begin{tabular}{|c|c|c|c|c|}
\hline Evaluation index & Index 1 & Index 2 & Index $n$ & Points \\
\hline \multicolumn{5}{|l|}{ Index 1} \\
\hline \multicolumn{5}{|l|}{ Index 2} \\
\hline$\vdots$ & & & & \\
\hline Index $n$ & & & & \\
\hline
\end{tabular}

Detailed instructions on how to fill in the form are listed as follows: Comparing row divisor with each column divisor, a very important index is given five points, a relatively important index is given four points, an equally important index is given three points, a not very important index is given two points, a not important index is given one point, and an non-essential index is given zero point. Finally we will get the total points of each index by accumulating each row points.

(3) Each expert is asked to fill in the questionnaire to obtain weighting factors, and the data is gathered as shown in Table 2.

Table 2 Statistics for weighting factors

\begin{tabular}{ccccc}
\hline Experts' name & Index 1 & Index 2 & $\ldots$ & Index $n$ \\
\hline Name 1 & $C_{11}$ & $C_{12}$ & $\ldots$ & $C_{1 n}$ \\
Name 2 & $C_{21}$ & $C_{22}$ & $\ldots$ & $C_{2 n}$ \\
$\vdots$ & $\vdots$ & $\vdots$ & $\vdots$ & $\vdots$ \\
Name $m$ & $C_{m 1}$ & $C_{m 2}$ & $\ldots$ & $C_{m n}$ \\
\hline
\end{tabular}

(4) Calculate the weight $\lambda$ for the evaluation index.

The weight $\lambda$ is calculated according to the following equation:

$$
\lambda_{j}=\frac{\frac{1}{m} \sum_{i=1}^{m} C_{i j}}{\sum_{j=1}^{n}\left(\frac{1}{m} \sum_{i=1}^{m} C_{i j}\right)}
$$

where $\lambda_{j}$ is the weight of $j$ th index; $C_{i j}$ is the score for the $j$ item evaluated by expert $i ; m$ is the number of experts participating in the evaluation; and $n$ is the total number of evaluated items.

\subsubsection{Questionnaire about risk grade}

A questionnaire on risk factor rankings, shown in Table 3 , is used to acquire risk evaluations from the experts for 
Table 3 Questionnaire on risk factor ranking

\begin{tabular}{|c|c|c|c|c|c|}
\hline Evaluation index & Minor risk & Medium risk & Greater risk & Fatal risk & Disaster risk \\
\hline
\end{tabular}

Index 1

Index 2

$\vdots$

Index $n$

each evaluation index for a specific ERW. The questionnaire includes risk evaluation indices (as mentioned in Fig. 1) and different risk grades (Grades 0-5) (Yong and Fu, 2002).

\section{The principles of comprehensive fuzzy evaluation}

The basic ideas of comprehensive fuzzy evaluation are as follows: (1) Fuzzify respective factors in various objects according to the evaluation criteria; (2) adopt fuzzy sets transform principle to construct the fuzzy evaluation matrix on the basis of determining the rating criteria and the weights of evaluation factors; (3) determine their ranks through multi-level complex computing ( $\mathrm{Li}$ and $\mathrm{Yu}, 2008$ ). The main procedure is as follows:

(1) Let $X$ be a set of all objects to be evaluated.

(2) Determine factor set of objects evaluated,

$X=\left\{x_{1}, x_{2}, \cdots, x_{\mathrm{m}}\right\}$

(3) Let $U$ be a set of reviews on objects,

$U=\left\{u_{1}, u_{2}, \cdots, u_{\mathrm{m}}\right\}$

$U$ is a full-ordered set, in which differences between any two remarks always exist. The typical sets of reviews are as follows: $U=$ (very good, good, general, poor, very poor), $U=$ (excellent, good, moderate, qualified, unqualified), $U=(1,2,3$, 4, 5 (point)), and $U=$ (A, B, C, D, E).

(4) Evaluate single factor to obtain evaluation vector of every factor and identify fuzzy evaluation matrix, expressed as follows:

$$
R=\left[\begin{array}{c}
R_{1} \\
R_{2} \\
\vdots \\
R_{n}
\end{array}\right]=\left[\begin{array}{cccc}
r_{11} & r_{12} & \cdots & r_{1 k} \\
r_{21} & r_{22} & \cdots & r_{2 k} \\
\vdots & \vdots & \vdots & \vdots \\
r_{n 1} & r_{n 2} & \cdots & r_{n k}
\end{array}\right]
$$

where $r_{i j}$ is membership degree, which can be obtained by selecting appropriate membership functions. For extended reach wells, $r_{i j}=y_{i j} / m$, where $m$ is the total number of experts participating in the evaluation and $y_{i j}$ is the number of experts who make factor $i$ belong to level $j$.

(5) Determine the weight vector matrix of single factor.

The weight vector matrix $W=\left[w_{1}, w_{2}, \cdots w_{m}\right], \sum_{i=1}^{m} w_{i}=1$ is mainly determined by the Analytic Hierarchy Process (AHP) and weight discrimination.

(6) Single-level comprehensive evaluation.

According to the single factor evaluation matrix and single factor weight vector matrix, a comprehensive fuzzy evaluation matrix $B$ can be obtained as follows: $B=W \cdot R$. If $U$ is quantified, the value of comprehensive fuzzy evaluation is calculated from $P=U \cdot B^{\mathrm{T}}$. The objects evaluated can be ranked according to the value of comprehensive fuzzy evaluations.

(7) Multi-level comprehensive evaluation.

From the bottom layer, a single-level comprehensive evaluation for inclusive factors is made and its evaluation result is defined as a row vector of fuzzy evaluation matrix of the upper layer. According to the vector weight and fuzzy evaluation matrix of the upper layer, the evaluation matrix of the uppermost layer is obtained.

(8) Convert comprehensive reviews into probability.

The evaluation results are usually expressed by membership degree vector and this expression method is not visual. Therefore, we generally take an arithmetic sequence to convert comprehensive reviews into risk probability or success probability.

\section{Applications}

The Liuhua 11-1 Oilfield, with a sea depth of $311 \mathrm{~m}$, is located in the Block 24/09 in the Eastern South China Sea, China. The oilfield was discovered in 1987 and put into production in March 29, 1996. The mega-extendedreach well development program was designed in the Well block 3 in the Liuhua Oilfield through a feasibility study, i.e. choosing an existing production well slot, using the existing production platform to sidetrack mega-extended-reach wells to produce from Well block 3 reservoir. This paper presents an 8,000-meter ERW with an ultra-high HD to VD ratio, Well B6ERW07 in Liuhua 11-1 Oilfield as an example, to analyze the drilling risk.

(1) Give Well B6ERW07 as an example to illustrate the risk evaluation of ERD.

(2) Risk factors of the model are denoted as the elements of the set of evaluation objects.

(3) The set of reviews on the objects $U=$ (minor risk, medium risk, greater risk, fatal risk, disaster risk).

(4) Determine the fuzzy evaluation matrix.

According to the principle of Section 3.2.2, the fuzzy 
evaluation matrix of a single factor was obtained. The fuzzy matrix of the risk of control before drilling and during drilling operation, $R_{1}$ and fuzzy matrix of the risk of equipment and its operation, $R_{2}$ are obtained from the risk questionnaire.

$$
\begin{aligned}
R_{1} & =\left[\begin{array}{ccccc}
0.0589 & 0.2941 & 0.3529 & 0.1765 & 0.1176 \\
0.4118 & 0.4706 & 0.1176 & 0 & 0 \\
0 & 0.0589 & 0.4706 & 0.1176 & 0.3529 \\
0 & 0.1765 & 0.4706 & 0.2353 & 0.1176 \\
0 & 0.1176 & 0.4706 & 0.3529 & 0.0589 \\
0.2353 & 0.5882 & 0.1765 & 0 & 0 \\
0.1176 & 0.2353 & 0.4706 & 0.1765 & 0 \\
0.0588 & 0.5294 & 0.2353 & 0.1765 & 0 \\
0.7059 & 0.2353 & 0.0588 & 0 & 0
\end{array}\right] \\
R_{2} & =\left[\begin{array}{ccccc}
0 & 0.3529 & 0.4706 & 0.1765 & 0 \\
0 & 0.1176 & 0.5883 & 0.1765 & 0.1176
\end{array}\right]
\end{aligned}
$$

(5) Determine the weights of risk evaluation indices.

According to the principle of Section 3.2.1, the weights of evaluation indices are obtained. The weights of the risk of control before drilling and during drilling operation in the bottom layer are as follows:

$$
\begin{aligned}
& W_{1}=\left[\begin{array}{llllll}
0.1201 & 0.0991 & 0.1432 & 0.1389 & 0.1279 & 0.0930
\end{array}\right. \\
& \left.\begin{array}{lll}
0.1283 & 0.0894 & 0.0601
\end{array}\right]
\end{aligned}
$$

The weights of the risk of equipment and its operation in the bottom layer are as follows:

$$
W_{2}=\left[\begin{array}{ll}
0.4146 & 0.5854
\end{array}\right]
$$

The weights of indices in the middle layer are as follows:

$$
W=\left[\begin{array}{ll}
0.5625 & 0.4375
\end{array}\right]
$$

(6) Single-level comprehensive fuzzy evaluation.

$$
\begin{aligned}
& B_{1}=W_{1} \cdot R_{1}=\left[\begin{array}{lllll}
0.1326 & 0.2763 & 0.3483 & 0.1543 & 0.0885
\end{array}\right] \\
& B_{2}=W_{2} \cdot R_{2}=\left[\begin{array}{lllll}
0 & 0.2152 & 0.5395 & 0.1765 & 0.0688
\end{array}\right]
\end{aligned}
$$

(7) Multi-level comprehensive fuzzy evaluation.

$$
R=\left[\begin{array}{ccccc}
0.1326 & 0.2763 & 0.3483 & 0.1543 & 0.0885 \\
0 & 0.2152 & 0.5395 & 0.1765 & 0.0688
\end{array}\right]
$$

Subordinated vector obtained finally is as follows:

$$
B=W \cdot R=\left[\begin{array}{lllll}
0.0746 & 0.2496 & 0.4319 & 0.1640 & 0.0799
\end{array}\right]
$$

According to the subordinated vector, the risk of drilling Well B6ERW07 is relatively high. An arithmetic sequence $U=(0,25,50,75,100)$ is used to convert comprehensive reviews into risk probability, $P=U \cdot B^{\mathrm{T}}=48.1$. So the probability of success is $51.9 \%$.

\section{Conclusions and suggestions}

1) The risk of drilling Well B6ERW07, an 8,000-meter ERW with a high HD to VD ratio, in the Liuhua Oilfield is relatively high and its probability of success is only about $51.9 \%$. Considerable difficulties exist in drilling and completing this well. Based on this research, the plan for drilling Well B6ERW07 has been postponed to the year 2010.

2) Comprehensive fuzzy evaluation is simple and easy to understand, and can deal well with complex multi-factor and multi-level problems. Comprehensive fuzzy evaluation method is practicable to evaluate the risk of extended reach wells.

3) Comprehensive fuzzy evaluation method can not only give qualitative analytical results, but also quantitative understanding through converting qualitative analytical results into the form of probability according to an arithmetic sequence.

\section{Acknowledgements}

The financial support from the project of CNOOC China Limited-Shenzhen (Grant No. Z2007SLSZ-034) and the foundation project of the State Key Laboratory of Petroleum Resource and Prospecting (Grant No. PRPDX2008-08) is gratefully acknowledged.

\section{References}

Jiang S Q. Developments of extended reach drilling and its implication. China Offshore Oil and Gas (Engineering). 1999. 11(3): 1-8, 14 (in Chinese)

Li Q and Yu L L. Integrated drilling risk evaluation method and model establishment. Natural Gas Industry. 2008. 28(5): 120-122 (in Chinese)

Modi S and Mason C J. Meeting the $10 \mathrm{~km}$ drilling challenge. SPE Annual Technical Conference and Exhibition, 5-8 October 1997, San Antonio, Texas (SPE 38583)

Wang D L. Oil Drilling Technology Development Planning and Research of Scientific and Technological Progress Evaluation. Beijing: Oil Industry Press. 2000. 1-31 (in Chinese)

Yong Q D and Fu J H. Risk assessment and analysis for extended reach drilling technique. Acta Petrolei Sinica. 2002. 23(1): 83-87 (in Chinese)

Zhang H and Gao D L. Selection method for oil \& gas drilling core technology. Drilling \& Production Technology. 2005. 29(1): 1-5 (in Chinese)

Zhang Y B and Zhang Y L. Qualitative forecast methods. Journal of Jilin Architectural and Civil Engineering Institute. 1999. 26(1): 59-62 (in Chinese)

(Edited by Sun Yanhua) 